\title{
The Tolosa-Hunt syndrome
}

\author{
L B Kline, W F Hoyt
}

\section{Defining the syndrome}

The syndrome of painful ophthalmoplegia consists of periorbital or hemicranial pain, combined with ipsilateral ocular motor nerve palsies, oculosympathetic paralysis, and sensory loss in the distribution of the ophthalmic and occasionally the maxillary division of the trigeminal nerve. Various combinations of these cranial nerve palsies may occur, localising the pathological process to the region of the cavernous sinus/superior orbital fissure.

The constellation of findings described may be due to four major causes: trauma, neoplasm, aneurysm, and inflammation. Comprehensive patient evaluation is essential in establishing the correct diagnosis.

Within the last diagnostic category there is a specific subset of patients who develop painful ophthalmoplegia due to a non-specific inflammatory process in the region of the cavernous sinus/superior orbital fissure. Infrequently, they experience involvement of additional cranial nerves ipsilateral to the ophthalmoplegia, including the optic nerve, mandibular branch of trigeminal nerve, and facial nerve. Having a relapsing and remitting course, they respond promptly to systemic corticosteroid therapy. The diagnostic eponym Tolosa-Hunt syndrome has been applied to these patients, ${ }^{1}$ and it is this entity which forms the basis of this review.

\section{Historical review}

Almost 50 years ago Tolosa ${ }^{2}$ reported a patient with left orbital pain, ipsilateral progressive visual loss, total left ophthalmoplegia, and reduced sensation over the first division of the trigeminal nerve. Cerebral angiography disclosed narrowing of the intracavernous segment of the left internal carotid artery. Surgical exploration of the left parasellar region was unremarkable, but the patient died 3 days later. At postmortem, granulomatous inflammation of the affected carotid artery and cavernous sinus was found.

Seven years later, Hunt et al defined a clinical entity "of somewhat obscure aetiology" on the basis of six patients. In one patient surgical exploration of the parasellar region showed all structures to be "intact and healthy". The authors thought that this syndrome of painful ophthalmoplegia was caused by an inflammatory lesion in the cavernous sinus, as described by Tolosa. ${ }^{2}$ They characterised it by the following diagnostic criteria:
(1) Pain may precede the ophthalmoplegia by several days, or may not appear until some time later. It is not a throbbing hemicrania occurring in paroxysms, but a steady pain behind the eye that is often described as "gnawing" or "boring".

(2) Neurological involvement is not confined to the third cranial nerve, but may include the fourth, sixth, and first division of the fifth cranial nerves. Periarterial sympathetic fibres and the optic nerve may be involved.

(3) Symptoms last for days to weeks.

(4) Spontaneous remissions occur, sometimes with residual neurological deficit.

(5) Attacks recur at intervals of months or years.

(6) Exhaustive studies, including angiography and surgical exploration, have produced no evidence of involvement of structures outside of the cavernous sinus. There is no systemic reaction.

In addition, Hunt and coworkers reported the therapeutic efficacy of systemic corticosteroids with prompt, dramatic improvement of signs and symptoms in two patients.

In 1966, Smith and Taxdal ${ }^{1}$ were the first to apply the eponym "Tolosa-Hunt syndrome" to this entity. They added five additional cases, "because there has been no previous report of this syndrome in the ophthalmology literature and to emphasise the use of steroids as a diagnostic test." The authors stated that "The administration of large doses of systemic steroids for 48 hours produces a dramatic response in painful ophthalmoplegia, which allows prompt differentiation of these cases."

Since these early descriptions of the TolosaHunt syndrome, numerous reports have confirmed these findings. The clinical syndrome has been found in virtually every continent of the world..$^{4-11}$ The non-specific nature of the pathological process has been confirmed, ${ }^{6}{ }^{12-14}$ as has the dramatic clinical response to systemic corticosteroids. ${ }^{510} 14$

However, over the past quarter century there has been no progress in understanding the pathogenesis of Tolosa-Hunt syndrome. In terms of clinical management some new information is available thanks to advances in neuroimaging techniques. Initially, radiological studies of patients with the syndrome yielded normal results, or subtle cerebral angiographic abnormalities. $^{2}{ }^{312} 15$ Orbital venography often disclosed abnormalities in filling of the superior ophthalmic vein or cavernous sinus, ${ }^{10}{ }^{16-19}$ but these techniques are difficult and therefore 
not consistently performed. In addition, venographic abnormalities are not specific for Tolosa-Hunt syndrome, and may be found with space occupying lesions or other inflammatory processes in the orbit or parasellar region. With the advent of CT and MRI direct visualisation of the cavernous sinus is possible. Using these neuroimaging modalities, cavernous sinus abnormalities have now been described in Tolosa-Hunt syndrome (see below).

Clinical profile of Tolosa-Hunt syndrome Tolosa-Hunt syndrome can affect people of virtually any age from the 1 st to the 8 th decades of life, with no sex predilection. Either side may be affected, with case reports of bilateral simultaneous involvement. Uniformly, patients complain of pain, which is a defining symptom. The pain lasts an average of 8 weeks if untreated. Ocular motor cranial nerve palsies may coincide with the onset of pain or follow it within a period of up to 2 weeks. ${ }^{20}$ It is usually described as "intense", "severe", "boring", "lancinating", or "stabbing". It is periorbital in location, frequently extending into the retroorbital, frontal, and temporal regions.

All three ocular motor cranial nerves may be involved, in various combinations. Pupillary reactions may be normal, or there may be either parasympathetic (oculomotor nerve) or sympathetic (third order neuron Horner's syndrome) involvement.

At times, optic nerve dysfunction has been reported with Tolosa-Hunt syndrome, indicating that the pathological process may involve the orbital apex. ${ }^{5-7} 2122$ The optic disc may be normal, swollen, or pale in appearance, and visual decline may be minimal or lead to blindness. Loss of acuity is variable and unpredictable, and, on occasion, may be permanent.

Infrequently, other cranial nerves not located within the cavernous sinus/superior orbital fissure may be affected. These include the maxil$\operatorname{lary}^{1617}$ and mandibular branch of the trigeminal nerve, ${ }^{623}$ and the facial nerve. ${ }^{1123}$

There are no reports of systemic or other neurological involvement in Tolosa-Hunt syndrome, with the exception of nausea and vomiting. These symptoms are probably due to the intense periorbital pain; they promptly resolve with cessation of pain.

\section{Natural history}

As Hunt outlined in his diagnostic criteria, ${ }^{3}$ Tolosa-Hunt syndrome follows an unpredictable course. Usually acute at onset, the patient's symptoms typically last from days to weeks. Before the use of systemic corticosteroids there was clear evidence that spontaneous remissions occurred. However, recurrences are common, taking place in about a half of reported patients, usually at an interval of months or years from the initial attack. ${ }^{124}$ These recurrences may be ipsilateral, contralateral, or rarely, bilateral.

Although Tolosa-Hunt syndrome is a self limited illness, it does cause considerable morbidity. Infrequently, residual cranial nerve palsies persist. With the institution of corticosteroid therapy, the natural course is altered.
Although there is no conclusive evidence to show that steroids lessen the degree or duration of the ophthalmoplegia, there is dramatic reduction of pain, often within 24 hours.

\section{Diagnostic evaluation}

With careful clinical examination, pain associated with typical cranial nerve palsies localises the pathological process to the regions of the cavernous sinus/superior orbital fissure. As noted above, current neuroimaging modalities allow visualisation of the area of suspected pathology. Contrast enhanced MRI with multiple views, particularly coronal sections, should be the initial diagnostic study performed. Numerous reports have demonstrated an area of abnormal soft tissue in the region of the cavernous sinus in most, but not all, patients with Tolosa-Hunt syndrome. ${ }^{25-29}$ Typically, the abnormality is seen as an intermediate signal intensity on $\mathrm{T} 1$ and intermediate weighted images, consistent with an inflammatory process. In addition, there is enhancement of the abnormal area after intravenous injection of paramagnetic contrast. With corticosteroid therapy, the abnormal area decreases in volume and signal intensity in most reported cases.

High resolution CT can also demonstrate soft tissue changes in the region of the cavernous sinus/superior orbital fissure, but is less sensitive than MRI. ${ }^{25}{ }^{30}$ This is due to lack of sensitivity to soft tissue change with superimposed beam hardening and bone streak artifacts. Thus, even if CT is normal, MRI must still be performed to appropriately evaluate the region of the cavernous sinus or superior orbital fissure.

The major limitation of MRI findings in Tolosa-Hunt syndrome is their lack of specificity. Thus, Yousem et al $l^{25}$ examined 11 patients and reported pathological MRI findings in the cavernous sinus in nine. In six of these nine the affected cavernous sinus was enlarged; in five of nine it had a convex lateral wall. Extension into the orbital apex was seen in eight patients. Yet the signal characteristics (hypointense relative to fat and isointense with muscle on $\mathrm{T} 1$ weighted images; isointense with fat on T2 weighted scans) were also consistent with meningioma, lymphoma, and sarcoidosis.

Because of this fundamental limitation of initial imaging studies, some authorities would suggest that resolution of imaging abnormalities after a course of systemic corticosteroids should be considered "diagnostic" of TolosaHunt syndrome. ${ }^{29}{ }^{31}$ However, caution is advised when assessing the salutary effects of steroids, as improvement both clinically and radiologically may occur with other disease processes. "False positive" steroid responsiveness with a "remitting" course can be seen with both malignant processes (for example, lymphoma) as well as more benign disorders (for example, vasculitis). ${ }^{20}$

Categorically, Tolosa-Hunt syndrome is a diagnosis of exclusion requiring careful patient evaluation (table 1) to rule out tumour, vascular causes, or other forms of inflammation in the region of the cavernous sinus/superior 
Table 1 Diagnostic evaluation of Tolosa-Hunt syndrome

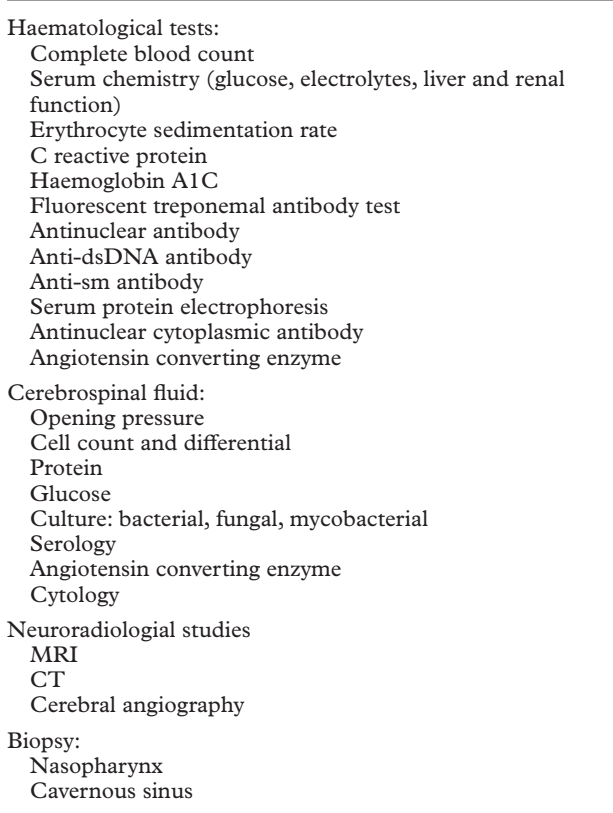

orbital fissure (see differential diagnosis below).

Occasional reports have documented a raised erythrocyte sedimentation rate and leukocytosis in the acute stage of Tolosa-Hunt syndrome. ${ }^{4-61722}$ Similarly, positive LE cell preparation and antinuclear antibody concentrations ${ }^{52}$ have been documented in patients with Tolosa-Hunt syndrome, but there is no convincing evidence that such patients either have or will develop connective tissue disorder.

In general, CSF examination should also be unremarkable, although rarely raised protein and mild pleocytosis have been reported. However, if CSF abnormalities persist, the diagnosis of Tolosa-Hunt syndrome is untenable, and further diagnostic evaluations are required.

As noted above, MRI is the initial diagnostic study to be performed in patients with a disorder of the cavernous sinus or superior orbital fissure. Computed tomography is a very useful adjunct in detecting bone changes (for example, erosion, hyperostosis) as well as (perisellar) calcification. Cerebral angiography has detected abnormalities in the intracavernous carotid artery in patients with Tolosa-Hunt syndrome..$^{5-7} 17182333$ These have been described as "segmental narrowing", "slight irregularity", or "constriction", and will resolve with corticosteroid therapy. The role of noninvasive vascular MRI techniques (MR angiography and venography) has not been defined in patients with the syndrome but these may prove to be valuable adjuncts in diagnostic evaluation.

Neurosurgical biopsy is only rarely employed to establish the diagnosis. ${ }^{5-7} 121733$ This can be technically difficult and should only be performed by experienced neurosurgeons. It usually involves biopsy of the dural wall of the cavernous sinus. Although generally a procedure of "last resort", it should be considered in patients with rapidly progressive neurological deficits, lack of steroid responsiveness, or persistent abnormalities on neuroimaging studies.

\section{Treatment}

Almost 40 years ago Hunt first documented the beneficial effect of corticosteroid therapy in Tolosa-Hunt syndrome. ${ }^{3}$ Unfortunately, since then there is little new information as to optimal dosage, duration of treatment, or alternative forms of therapy. It is clear that spontaneous remissions may occur, but there is no doubt that corticosteroids markedly reduce the periorbital pain. No data are available as to whether treatment hastens recovery of the associated cranial nerve palsies. Although steroids are generally tapered over weeks to months, in some cases prolonged therapy may be necessary. As cautioned previously, positive response to steroids has been reported in various parasellar neoplasms, including chordoma, giant cell tumour, lymphoma, and epidermoid. $^{24}$

\section{Pathology}

Tolosa-Hunt syndrome is caused by an inflammatory process. Tolosa ${ }^{2}$ originally described non-specific, chronic inflammation with proliferation of fibroblasts and infiltration of the septa and wall of the cavernous sinus with lymphocytes and plasma cells. Hunt et al corroborated these findings, emphasising the lack of necrosis and pointed out that " ... such inflammatory changes, in a tight connective tissue, may exert pressure upon the penetrating nerves." Subsequent reports have shown granulomatous inflammation, with epithelioid cells and occasional giant cells. ${ }^{6}{ }^{12}$ Necrosis may also be seen. There have been no reports of an infectious organism associated with Tolosa-Hunt syndrome.

\section{Aetiology}

The aetiology of Tolosa-Hunt syndrome remains unknown. No information is available as to what triggers the inflammatory process in the region of the cavernous sinus/superior orbital fissure. It seems that the syndrome falls within the range of idiopathic orbital inflammation (pseudotumour). ${ }^{34}$ Thus, "nonspecific" inflammation typically causes an acute orbitopathy. If located more posteriorly, the inflammatory process involves the cavernous sinus/superior orbital fissure, producing the Tolosa-Hunt syndrome. Rarely, the inflammation may spread intracranially. ${ }^{35-37}$

\section{Differential diagnosis}

Because the diagnosis of Tolosa-Hunt syndrome can been made only after other disease processes have been excluded, it behoves the clinician to be familiar with the differential diagnosis of painful ophthalmoplegia. In fact, during the initial patient evaluation there are often no clues in the history or physical examination to distinguish Tolosa-Hunt syndrome from other causes of painful ophthalmoplegia. Therefore, the clinician should be aware of: (1) causes of parasellar syndrome and (2) other entities producing painful ophthalmoplegia. 
Table 2 Causes of parasellar syndrome producing painful ophthalmoplegia

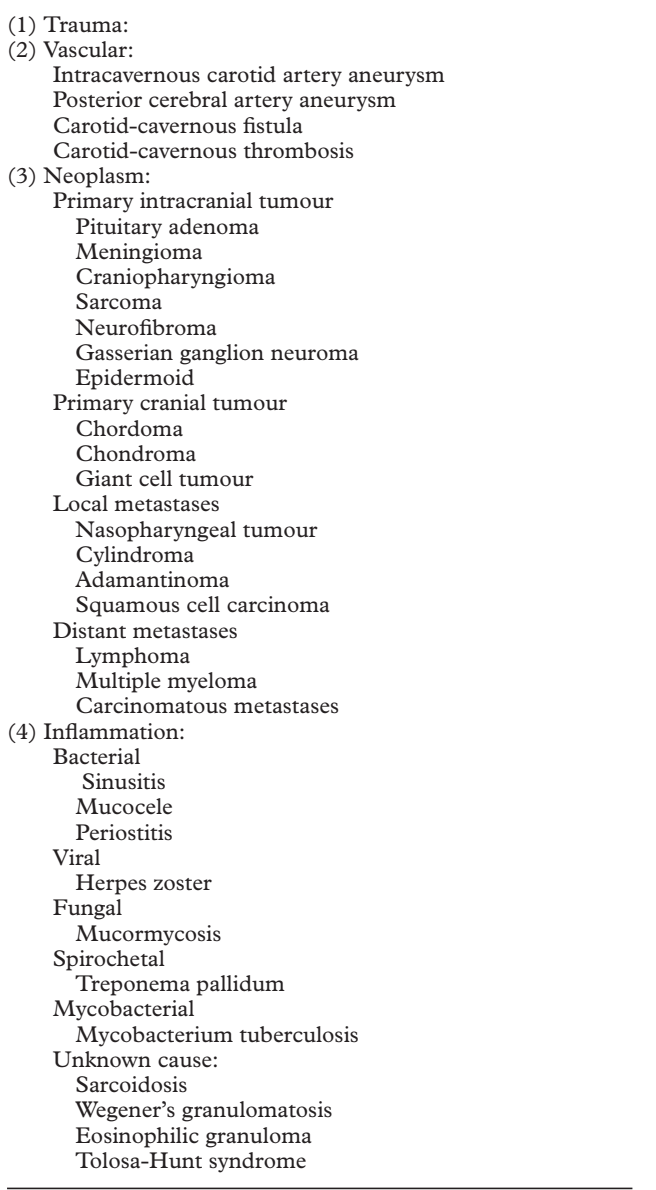

CAUSES OF PARASELLAR SYNDROME (TABLE 2) Craniocerebral trauma may produce painful ophthalmoplegia in various ways: basilar skull fracture with ocular motor nerve damage, intracavernous carotid artery injury with subsequent aneurysm formation, or carotidcavernous fistula.

Various vascular causes may produce painful ophthalmoplegia, the most frequent being an intracavernous carotid artery aneurysm. ${ }^{38} 39$ Rarely, giant aneurysms of the posterior circulation, including the posterior cerebral artery, may mimic Tolosa-Hunt syndrome. ${ }^{40}$ Carotidcavernous fistula and cavernous sinus thrombosis also have acute onset of symptoms and signs, and must be included in the differential diagnosis. Patients with carotid-cavernous fistulae rarely complain of severe pain and typically have dramatic ophthalmological signs including proptosis, "arterialised" conjunctival vessels, chemosis, increased intraocular pressure, and retinal vascular abnormalities. A bruit is often present, cerebral angiography is the diagnostic procedure of choice, and interventional angiographic techniques are usually curative. Cavernous sinus thrombosis may be septic or aseptic in origin. In the first, there may be associated signs of sinusitis, otitis, gingivitis, or orbital cellulitis. Patients are febrile, have a leukocytosis, and if the infectious process spreads intracranially, seizures and altered mental states may occur. Aseptic cavernous sinus thrombosis may be associated with various conditions including polycythemia, sickle cell disease, vasculitis, pregnancy, dehydration, trauma, and intracranial surgery. Regardless of aetiology, cavernous sinus thrombosis is characterised by orbital congestion, proptosis, eyelid swelling, chemosis, lacrimation, and ophthalmoparesis. Pain around or behind the eye is common. Treatment of cavernous sinus thrombosis usually involves anticoagulant drugs, at times thrombolytic agents, and in septic cases, appropriate antibiotic therapy.

As outlined in table 2, painful ophthalmoplegia may be caused by either contiguous or metastatic spread of a neoplasm. Metastatic involvement of the cavernous sinus/superior orbital fissure is generally due to haematogenous dissemination of neoplastic cells. ${ }^{41}$ Occasionally, intracranial neoplastic invasion may occur by intraneural or perineural spread, as in the case of squamous cell carcinoma producing painful ophthalmoplegia many months after local excision of a facial skin tumour. ${ }^{42}$

Two aspects of neoplastic involvement of the cavernous sinus/superior orbital fissure require emphasis. Firstly, it is important to realise that mode of onset and clinical course are not indicative of the type of lesion causing painful ophthalmoplegia. In their review of parasellar syndrome, Thomas and Yoss" ${ }^{43}$ state that "A sudden onset of symptoms does not weigh in favor of aneurysmal or against neoplastic origin, and gradual onset is not necessarily indicative of a neoplasm ... spontaneous single or multiple remissions of symptoms, even of years duration, are unreliable in predicting the nature of the underlying process." Secondly, as noted above, high dose corticosteroid therapy may initially improve signs and symptoms due to neoplasm.

Inflammatory causes of painful ophthalmoplegia include those due to a specific infectious agent. It is essential that careful CSF examination be done and that cultures (bacterial, fungal, mycobacterial) be obtained. The potential role of a paranasal sinus as a cause of painful ophthalmoplegia requires attention. Sinus disease may lead to cavernous sinus involvement, either via contiguous spread of infection or due to sphenoid sinus mucocele.

OTHER CAUSES OF PAINFUL OPHTHALMOPLEGIA Table 3 summarises other aetiologies of painful ophthalmoplegia in which there is no involvement of the cavernous sinus/superior orbital fissure.

Various orbital diseases cause painful ophthalmoplegia. Typically, the patient presents with "orbital signs", including proptosis, conjunctival injection, chemosis, and resistance to retrodisplacement of the globe. In addition, the eye may be displaced within the orbit, and there may be abnormalities of the ocular adnexa (for example, lids, lacrimal gland).

Diabetic ophthalmoplegia typically produces an acute, often painful mononeuropathy in either a known or previously undiagnosed diabetic person. Invariably there is recovery of ocular motor cranial nerve function, usually 
Table 3 Additional causes of painful ophthalmoplegia

Orbital disease:

Idiopathic orbital inflammation (pseudotumour)

Contiguous sinusitis

Mucormycosis or other fungal infection

Metastatic tumour

Lymphoma/leukaemia

Diabetic ophthalmoplegia:

Mononeuropathy

Multiple cranial nerve palsies

Posterior fossa aneurysm:

Posterior communicating artery

Basilar artery

Giant cell arteritis:

Ophthalmoplegic migraine:

within 3 months. In addition, there are reports of diabetic patients with simultaneous paralysis of multiple ocular motor nerves. ${ }^{45}$ Frequently, these episodes are painful, often recurrent, and not particularly responsive to corticosteroid therapy.

Posterior fossa aneurysms may produce either acute, painful ophthalmoplegia, or may present in a more subacute or chronic fashion. The acute presentation is most often due to an aneurysm in the anterior circulation, typically at the junction of the internal carotid-posterior communicating arteries, whereas the subacute presentation is caused by basilar artery aneurysms. In both, cerebral angiography is diagnostic.

Giant cell arteritis may also produce painful ophthalmoplegia. The clinical picture may be one of single or multiple ocular motor nerve palsies. In the only pathological study of ophthalmoplegia occurring in giant cell arteritis, ischaemic necrosis of the extraocular muscles was demonstrated. Examination of the ocular and motor cranial nerves was unremarkable. $^{46}$

Ophthalmoplegic migraine is a poorly understood form of complicated migraine. ${ }^{47}$ Typically, this clinical syndrome occurs in a child or young adult with periodic headache, who develops an ocular motor cranial nerve palsy at the height of an attack of cephalgia, which is primarily unilateral and in the orbital region. Most often involved is the oculomotor nerve, occasionally the abducens nerve, and rarely the trochlear nerve. The paresis lasts for days to weeks after cessation of a headache; recovery is gradual and tends to be less complete after repeated attacks. A family history of migraine is usually obtained. There are reports of enhancement of the extra-axial portion of the oculomotor nerves of patients with ophthalmoplegic migraine when evaluated with contrast-enhanced MRI. ${ }^{48-50}$

\section{Conclusion}

Although the pathogenetic basis of TolosaHunt syndrome remains unknown, from a practical clinical standpoint it can be regarded as a distinct entity which may be simulated by various other disorders. It cannot be emphasised too strongly that patients suspected of having the syndrome require careful evaluation, appropriate treatment, and scrupulous follow up observation.
The work is supported by an unrestricted grant from Research to Prevent Blindness Inc, New York, USA.

1 Smith JL, Taxdal DSR. Painful ophthalmoplegia. the Tolosa-Hunt 72 .

2 Tolosa E. Periarteritic lesions of the carotid siphon with the clinical features of a carotid infraclinoid aneurysm. $\mathcal{F} \mathrm{Neu}-$ rol Neurosurg Psychiatry 1954;17:300-2.

3 Hunt WE, Meagher JN, Le Fever HE, et al. Painful ophthalmoplegia. Its relation to indolent inflammation of the cavernous sinus. Neurology 1961;11:56-62.

4 Lakke JPWF. Superior orbital fissure syndrome. Report of a case caused by local pachymeningitis. Arch Neurol 1962;7: 289-300.

5 Mathew NT, Chandy J. Painful ophthalmoplegia. $\mathcal{F}$ Neurol Sci 1970;11:243-56.

6 Schatz NJ, Farmer P: Tolosa-Hunt syndrome: the pathology of painful ophthalmoplegia. In: Smith JL, ed. Neuroophthalmology. Symposium of the University of Miami and the ophthalmology. Symposium of the University of Miami and the 102-12.

7 Spinnler H. Painful ophthalmoplegia; the Tolosa-Hunt syndrome. Med F A A st 1973;2:645-6.

8 Roca PD. Painful ophthalmoplegia: the Tolosa-Hunt syndrome. Ann Ophthalmol 1975;7:828-34.

9 Cohn DF, Carasso R, Streifler M. Painful ophthalmoplegia. Eur Neurol 1979;18:373-81.

10 Muhletaler CA, Gerlock AJ. Orbital venography in painful ophthalmoplegia (Tolosa-Hunt syndrome). Am $\mathcal{f}$ Radiol 1979;133:31-4.

11 Inzafari D, Sita D, Marconi GP, et al. The Tolosa-Hunt syndrome: further clinical and pathogenetic considerations based on the study of eight cases. F Neurol 1981;224:221-8.

12 Aron-Rosa D, Doyon D, Sallamon G, et al. Tolosa-Hunt syndrome. Ann Ophthalmol 1978;10:1161-8.

13 Goadsby PJ, Lance JW. Clinicopathological correlation in a case of painful ophthalmoplegia: Tolosa-Hunt syndrome. $\mathcal{F}$ Neurol Neurosurg Psychiatry 1989;52:1290-3.

14 Hunt WE. Tolosa-Hunt syndrome: one cause of painful ophthalmoplegia. F Neurosurg 1976;44:544-9.

15 Van Dalen JTW, Blecker GM. The Tolosa-Hunt syndrome. Doc Ophthalmol 1977;44:167-72.

16 Milstein BA, Morretia LB. Report of a case of sphenoid fissure syndrome studied by orbital venography. Am $\mathcal{7}$ Ophthalmol 1971;72:600-3.

17 Sondheimer FK, Knapp J. Angiographic findings in the Tolosa-Hunt syndrome: painful ophthalmoplegia. Radiology 1973;106:105-12.

18 Julien J, Vallat JM, Vallat M, et al. Orbital phlebography in the diagnosis of painful ophthalmoplegia. Neuroradiology the diagnosis of

19 Hannetz J. Recurrent Tolosa-Hunt syndrome: a report of 10 new cases. Cephalgia 1999;19 (suppl 25):33-5.

20 Förderreuther S, Straube A. The criteria of the International Headache Society for Tolosa-Hunt syndrome need to be revised. Neurol 1999;246:371-7.

21 Anderson BI. Unusual course of painful ophthalmoplegia. Report of a case. Acta Ophthalmol 1980;58:841-8.

22 Zafrulla KM, Dhir SP, Jain IS. Tolosa-Hunt syndrome. Ind f Ophthalmol 1977;25:21-6.

23 Adams AH, Warner AW. Painful ophthalmoplegia: report of a case with cerebral involvement and psychiatric complications. Bull Los Angeles Neurol Soc 1975;40:49-55.

24 Kline LB. The Tolosa-Hunt syndrome. Surv Ophthalmol 1982;27:79-95.

25 Yousem DM, Atlas SW, Grossman RI, et al. MR imaging of Tolosa-Hunt syndrome. AfNR Am f Neuroradiol 1990;10: $1181-4$.

26 Gato Y, Hosokawa S, Goto I, et al. Abnormality in the cavernous sinus in three patients with Tolosa-Hunt syndrome: MRI and CT findings. F Neurol Neurosurg Psychiatry 1990; 53:231-4

27 Desai SP, Carter J, Jinkins JR. Contrast-enhanced MR imaging of Tolosa-Hunt syndrome: a case report. AfNR Am F Neuroradiol 1991;12:182-3.

28 Obadasi Z, Gökeil Z, Atilla S, et al. The value of MRI in a case of Tolosa-Hunt syndrome. Clin Neurol Neurosurg 1997;99:151-4

29 Areaya AA, Cerezal L, Canga A, et al. Neuroimaging diagnosis of Tolosa-Hunt syndrome: MRI contribution. Headache 1999;39:321-5.

30 Kwan ESK, Walpert SM, Hedges TR, et al. Tolosa-Hunt revisited: not necessarily a diagnosis of exclusion. AfNR revisited: not necessarily a diagnosis

31 Pascual J, Cerezal L, Canga A, et al. Tolosa-Hunt syndrome: focus on MRI diagnosis. Cephalgia 1999;19(suppl 25): $36-8$

32 Lesser R, Jampol LM. Tolosa-Hunt syndrome and antinuclear factor. Am f Ophthalmol 1974;77:732-4.

33 Hallpike JF. Superior orbital fissure syndrome. 7 Neurol Neurosurg Psychiatry 1973;36:486-90.

34 Levy IS, Wright JE, Lloyd GAS. Orbital and retro-orbital pseudo-tumor. Modern Problems of Ophthalmology 1975;14: 364-7.

35 Kaye AH, Hahn JF, Craciun A, et al. Intracranial extension of inflammatory pseudotumor of the orbit. $f$ Neurosurg 1984;60:625-9.

36 Frohman LP, Kupersmith MJ, Lang J, et al. Intracranial extension and bone destruction in orbital pseudotumor. Arch Ophthalmol 1986;104:380-4. 
37 Bencheif B, Zovaoui A, Chedid G, et al. Intracranial extension of idiopathic orbital inflammatory pseudotumor. sion of idiopathic orbital inflammat
AFNR Am $\mathcal{7}$ Neuroradiol 1993;14:181-4.

38 Meadows SP. Intracavernous aneurysms of the internal carotid artery. Arch Ophthalmol 1959;65:566-74.

39 Trobe JD, Glaser JS, Post MJD. Meningiomas and aneurysms of the cavernous sinus. Arch Ophthalmol 1978;96:457-67.

40 Coppedo JR, Hoffman H. Tolosa-Hunt syndrome with proptosis mimicked by giant aneurysm of the posterior cerebral artery. Arch Neurol 1981;38:54-5.

41 Post MJD, Mendez DR, Kline LB, et al. Metastatic disease to the cavernous sinus. F Comput Assist Tomogr 1985;9:11520.

42 Trobe JD, Hood CI, Parsons JT, et al. Intracranial spread of squamous carcinoma along the trigeminal nerve. Arch Ophthalmol 1982;100:608-11.

43 Thomas JE, Yoss RE. The parasellar syndrome: problems in determining etiology. Mayo Clin Proc 1978;45:617-23.
44 Larson DL, Auchincloss JH. Multiple symmetric bilateral cranial nerve palsies in patients with unregulated diabetes mellitus: report of three cases. Ann Intern Med 1950;85:

45 Eshbaugh CG, Siatkowski RM, Smith JL, et al. Simultaneous, multiple cranial neuropathies in diabetes mellitus. $\mathcal{F}$ Neuroophthalmol 1995;15:219-24.

46 Barricks ME, Traviesa DB, Glaser JS, et al. Ophthalmoplegia in cranial arteritis. Brain 1977;100:209-21.

47 Friedman AP, Hunter GH, Merritt HH. Ophthalmoplegic migraine. Arch Neurol 1962;7:320-7.

48 Mark AS, Blake P, Atlas SW, et al. Gd-DTPA enhancement of the cisternal portion of the oculomotor nerve on MR imaging. AfNR Am f Neuroradiol 1992;13:1463-70.

49 Stommel EW, Ward TN, Harris RD. MRI findings in a case of ophthalmoplegic migraine. Headache 1993;33:234-7.

50 Østergaard JR, Moller HV, Christensen T. Recurrent ophthalmoplegia in childhood: diagnostic and etiologic

\section{What's in the next issue}

\section{Future content}

See which articles have just been accepted for publication and preview the table of contents for the next issue a month before it is published

www.jnnp.com 